\title{
Special announcement: Guidelines to the Practice of Anesthesia, Revised Edition 2014
}

\author{
Richard N. Merchant, MD • Matt M. Kurrek, MD
}

Received: 7 October 2013/Accepted: 18 October 2013/Published online: 3 January 2014

(C) Canadian Anesthesiologists' Society 2013

This issue of the Canadian Journal of Anesthesia (the Journal) continues its policy of publishing the yearly revised version of the Guidelines to the Practice of Anesthesia $^{1}$ (the Guidelines) as developed by the Committee on Standards and approved by the Board of the Canadian Anesthesiologists' Society (CAS). Initially developed in 1974 and published in 1975, the Guidelines are updated regularly and the revisions are published annually. This is now the fifth year in which the Guidelines are being published as a special article within the Journal.

The Committee continuously reviews features of the Guidelines and responds to the changing practice of anesthesiology with updates to the recommendations. In 1988, the following view was expressed in an editorial in the Journal, "In developing the guidelines the society has attempted to achieve a consensus as to what is practical, realistic and acceptable by a broad sample of concerned physicians. The statements cannot be based upon facts or proof because there are none - one can only go with the best information available. It is emphasized that guidelines can encourage high-quality patient care, but by no means do they guarantee any specific patient outcome." The forgoing perspective remains largely true today, though we consider the strength of evidence to be stronger in 2014 than in 1988. This year, the Guidelines incorporate three significant recommendations regarding important areas of anesthesia practice in Canada.

The first involves subspecialty areas of anesthesia practice. A broad review was undertaken in concert with

R. N. Merchant, MD $(\bowtie) \cdot$ M. M. Kurrek, MD

Committee on Standards, Canadian Anesthesiologists' Society,

1 Eglinton Avenue East, Suite 208, Toronto, ON M4P 3A1,

Canada

e-mail: richard.merchant@ubc.ca representatives from the CAS Pediatric Anesthesia group to ensure the Guidelines include wording that provides appropriate guidance for pediatric anesthesia. The pediatric anesthesia population includes children ranging in age from the neonatal (perhaps even pre-natal) period to the "near-adult" age of 19 years. Within this population, there are specific groups of children at the extremes of the age range as well as those with special comorbidities undergoing procedures of variable complexity. We have identified areas where anesthesia departments must develop policies and procedures - while considering their members' specific training and experience in pediatric anesthesia and the situations in which they practice-in order to care for such challenging patients in a safe and effective manner.

The second significant recommendation relates to patient care perhaps at the other extreme of care: i.e., in adult hospitals where our practice involves patients near or at the very limit of life. In these patients, proposed intervention may lead to events culminating in management decisions that may not be in keeping with patients' wishes and may be unbeknownst to their care providers. Respect for the principles of autonomy, informed consent, dignity, and prevention of suffering necessitate that discussions regarding "advance care planning", "living wills", or "do not resuscitate status" should be considered prior to embarking on an intervention. Without such thoughtful discussion, we miss the opportunity to honour these fundamental principles. As consultants, anesthesiologists share in the "most responsible physician" role in perioperative care; it is not reasonable to leave such assessments solely to the vagaries of surgical care. Anesthesiologists must be proactive in ensuring that the proposed care is in accordance with the wishes of the patient. Wording to this effect is added in the "Preoperative Care" section of the Guidelines. 
Lastly, the issue of "fatigue" or "fitness to practice" has been before the Committee on Standards for many years; indeed, a comment on this topic written by the Chair of the CAS Committee on Standards was published in the Journal in $1987 .^{3}$ This topic has been difficult to address because of the large variety of practices-from the smallest to the largest communities-in which anesthesia care is provided in Canada. To precisely define 'clinician fitness' in clinical situations is awkward, which is partly why the development of guidelines addressing this issue has been slow to evolve.

There is now irrefutable evidence, in both lay and professional literature, that truly acceptable care is provided only by practitioners who are optimally "fit" (i.e., medically and psychologically well and optimally rested) to practice. In this context, departments of anesthesia must implement policies and guidelines which are consistent with their particular circumstances and facilitate optimal care for their patients.

Reviewers and consultants such as regulatory review bodies and patient safety advocates will be assertive in defining this. Anesthesiologists should be relieved of their clinical duties in situations of significant illness or communicable disease. For example, an anesthesiologist with a minor upper respiratory tract infection may be able to work, but one requiring medication that may impact cognitive abilities or one suffering from infectious influenza should not be in a setting with exposure to the public. Significant psychological stress can also make a clinician unable to provide appropriate care, and departments must develop policies to manage such circumstances (e.g., an individual who has suffered a major intraoperative catastrophe should be relieved from duties for the day). Overly long work hours combined with inadequate rest raises the issue of fatigue, a topic that continues to receive considerable attention because of highprofile accidents where fatigue has been identified as at least a compounding factor. The public's need for $24 / 7$ anesthesia services, together with the realities of practice in remote areas, makes it difficult to address this problem through a "one-size-fits-all" recommendation. Often physicians themselves contribute to the problem by electing to work long hours, a mind-set generally triggered by a fee-forservice environment that links long work hours to higher personal income. If primary anesthesia care regularly requires excessively long clinical shifts, the expectation is that the clinical workload will be shared with other members. In circumstances where long hours of care are required (e.g., an overnight shift), a significant break (eight hours has been suggested) from clinical care is advised before the anesthesiologist undertakes any further elective care.

In light of these important concerns, the Guidelines stipulate that departments of anesthesia develop policies to ensure, insofar as is possible, that their patients receive the best possible care from practitioners optimally able to provide such care. Also, in recommending these Guidelines, the CAS recognizes that implementing change from the status quo may require a period of transition along with innovative and creative departmental strategies. Nevertheless, considering that our specialty promotes vigilance and safety, the issue of working while unwell or fatigued can no longer be ignored.

The CAS Committee on Standards and partner groups within the CAS actively continue to consider these and other pertinent issues. The Committee welcomes any feedback that members of the Canadian anesthesia community wish to provide and plans to develop formal mechanisms for such community involvement in the future.

\section{Annonce spéciale: Guide d'exercice de l'anesthésie, édition révisée 2014}

Dans ce numéro, le Journal canadien d'anesthésie (le Journal) poursuit sa politique de publication de la version, révisée annuellement, du Guide d'exercice de l'anesthésie ${ }^{1}$ (le Guide) tel que mis au point par le Comité des normes de pratique et approuvé par le Conseil d'administration de la Société canadienne des anesthésiologistes (SCA). Le Guide, élaboré pour la première fois en 1974 et publié en 1975, est régulièrement mis à jour et une version révisée est publiée chaque année. Voici la cinquième année que le Guide est publié sous forme d'article spécial dans le Journal.

Le Comité passe continuellement en revue certains aspects du Guide et répond à l'évolution de la pratique de l'anesthésiologie en mettant à jour les directives. En 1988, l'opinion suivante était exprimée dans un éditorial du Journal: ${ }^{2}$ "En mettant au point ce Guide, l'objectif de la Société était de parvenir à un consensus quant à ce qui est pratique, réaliste et acceptable pour un large échantillon des médecins concernés. Les énoncés ne peuvent se fonder sur des faits ou des preuves parce qu'il n'y en a pas - nous ne pouvons que nous fonder sur les meilleures informations à notre disposition. Nous tenons à souligner qu'un tel guide peut encourager des soins aux patients de qualité élevée; toutefois, il ne peut en aucun cas garantir un pronostic spécifique. " L'opinion exprimée ici demeure d'actualité en grande partie - bien que, selon nous, les données probantes soient plus fiables aujourd'hui en 2014 qu'elles ne l'étaient en 1988. Cette année, le Guide intègre trois directives d'importance concernant des domaines cruciaux de la pratique de l'anesthésie au Canada. 
La première touche aux domaines de surspécialité de la pratique de l'anesthésie. Une importante révision a été entreprise, de concert avec des représentants du groupe d'anesthésie pédiatrique de la SCA, afin de s'assurer que la formulation dans le Guide soit adéquate à guider l'anesthésie pédiatrique. La population touchée par l'anesthésie pédiatrique comprend des enfants dont l'âge varie de la période néonatale (voire prénatale) à un âge approchant celui d'un adulte, soit 19 ans. Au sein de cette population, il existe des groupes spécifiques d'enfants aux extrêmes de l'âge ainsi que d'autres présentant des comorbidités particulières et subissant des interventions de difficulté variable. Nous avons identifié les domaines dans lesquels les départements d'anesthésie doivent mettre au point des politiques et des procédures, tout en tenant compte de la formation et de l'expérience spécifique de leurs membres en matière d'anesthésie pédiatrique et des contextes dans lesquels ils pratiquent, afin de prendre soin de ces patients aux besoins particuliers de façon sécuritaire et efficace.

La deuxième recommandation importante est liée aux soins des patients se trouvant peut-être à l'autre extrême du spectre des soins, c'est-à-dire dans des hôpitaux pour adultes où notre pratique touche des patients à la limite de la vie, ou très proches de cette limite. Chez ces patients, l'intervention proposée pourrait mener à des événements culminant en décisions de prise en charge qui pourraient ne pas s'aligner avec les souhaits des patients et pourraient ne pas être connues des personnes qui en prennent soin. Si l'on souhaite respecter les principes d'autonomie, de consentement éclairé, de dignité et de prévention de la souffrance, il faut envisager d'avoir des discussions concernant un «plan de soins avancés », les «testaments biologiques» ou «l'état ne pas réanimer» avant d'entreprendre toute intervention. Si une telle discussion réfléchie n'est pas amorcée, nous manquons l'occasion d'honorer ces principes fondamentaux. En tant que consultants, les anesthésiologistes tiennent eux aussi le rôle du « médecin le plus responsable » en matière de soins périopératoires; il n'est en effet pas raisonnable de laisser de telles évaluations uniquement aux aléas des soins chirurgicaux. Les anesthésiologistes se doivent d'être proactifs pour garantir que les soins proposés s'alignent avec les souhaits du patient. Un énoncé à cet effet a été ajouté dans la section des «Soins préopératoires» du Guide.

Enfin, la question de la « fatigue » et de «l'aptitude au travail » préoccupe le Comité des normes de pratique depuis de nombreuses années; un commentaire du président du Comité des normes de pratique de la SCA avait déjà été publié à ce sujet dans le Journal en $1987 .{ }^{3} \mathrm{Ce}$ sujet a été difficile à aborder en raison de la diversité de pratique - de la collectivité la plus petite à la plus importante - des soins d'anesthésie au Canada. Il est malaisé de définir précisément ce qu'on entend par «l'aptitude du clinicien » dans des situations cliniques; c'est en partie pour cela que la mise au point de directives abordant cette question a été lente.

Il existe aujourd'hui des données irréfutables, tant dans la littérature profane que dans la littérature professionnelle, selon lesquelles des soins véritablement acceptables ne peuvent être prodigués que par des praticiens qui sont «aptes », de façon optimale (c.-à-d. en bonne santé médicale et psychologique et bien reposés), à pratiquer. Ainsi, les départements d'anesthésie doivent mettre en œuvre des politiques et des directives qui tiennent compte de leurs circonstances particulières et facilitent la fourniture de soins optimaux à leurs patients.

Les évaluateurs et les consultants, notamment ceux des organismes de réglementation et de défense de la sécurité des patients, seront très affirmatifs dans la définition de l'aptitude à la pratique. Les anesthésiologistes doivent être relevés de leurs tâches cliniques dans les situations de maladie grave ou de maladie contagieuse. Par exemple, un anesthésiologiste souffrant d'une infection des voies respiratoires supérieures mineure peut être apte à travailler, mais un anesthésiologiste devant prendre des médicaments qui pourraient affecter ses capacités cognitives ou souffrant d'influenza infectieuse ne devrait pas se trouver dans un contexte où il serait exposé au public. Un important stress psychologique peut également rendre un clinicien inapte à prodiguer des soins adaptés, et les départements doivent mettre en place des politiques pour gérer de telles circonstances (par ex., un individu ayant vécu une catastrophe peropératoire majeure devrait être relevé de ses fonctions pour la journée). Les heures de travail trop longues, en conjonction avec un repos inadapté, soulèvent la question de la fatigue, un sujet qui continue d'attirer une attention considérable en raison d'accidents très publicisés dans lesquels la fatigue a joué un rôle, du moins en tant que facteur aggravant. Le besoin du public de services d'anesthésie 24 h sur 24, sept jours par semaine, associé aux réalités de la pratique dans les régions éloignées, rend le problème difficile à résoudre avec une solution applicable à toutes les situations. Il arrive souvent que les médecins contribuent eux-mêmes au problème en choisissant de travailler de longues heures, une mentalité généralement motivée par un environnement de paiement à l'acte qui fait correspondre de longues heures de travail à des revenus personnels plus élevés. $\mathrm{Si}$ les soins d'anesthésie primaires nécessitent souvent des quarts de travail clinique excessivement longs, l'attente est que la charge de travail clinique soit partagée avec d'autres membres du département. Dans les cas où de longues heures de soins sont nécessaires (par ex. un quart de nuit), on recommande d'accorder une pause conséquente (on a 
suggéré huit heures) des soins cliniques avant que l'anesthésiologiste n'entreprenne d'autres soins non urgents.

À la lumière de ces préoccupations importantes, le Guide stipule que les départements d'anesthésie doivent mettre au point des politiques afin de garantir - dans la mesure du possible - que leurs patients reçoivent les meilleurs soins possibles de praticiens les plus aptes qui soient à prodiguer de tels soins. En outre, en appuyant ce Guide, la SCA reconnaît que la mise en œuvre de changements à partir du statu quo pourrait nécessiter une période de transition ainsi que des stratégies départementales innovatrices et créatives. Toutefois, étant donné que notre spécialité promeut la vigilance et la sécurité, la question du travail lorsqu'on n'est pas en pleine forme ne peut plus être ignorée.

Le Comité des normes de pratique de la SCA et les groupes partenaires au sein de la SCA continuent de réfléchir activement à ces questions ainsi qu'à d'autres questions pertinentes. Le Comité apprécie tout commentaire que les membres que la communauté anesthésique canadienne aurait. En outre, elle a l'intention de mettre en place des mécanismes formels afin de faciliter l'engagement de la communauté à l'avenir.

Competing interests None declared.

Conflit d'intérêt Aucun déclaré.

\section{References}

1. Merchant R, Chartrand D, Dain S, et al. Guidelines to the Practice of Anesthesia Revised Edition 2014. Can J Anesth 2014; 61: this issue.

2. Anonymous. Standards of practice - the Canadian experience. Can J Anaesth 1988, 35: 283-5.

3. Sheffman EJ. Revised Guidelines to the Practice of Anaesthesia. Can J Anaesth 1987; 34: 539. 